\title{
Prone Transpsoas Approach for Adjacent Segment Disease and Flatback Deformity: Technical Note and Case Report
}

\author{
Timothy E. O'Connor'1,2, Mary Margaret O’Hehir³, Jennifer Z. Mao², Jeffrey P. Mullin'1, \\ John Pollina ${ }^{1,2 *}$ \\ ${ }^{1}$ Department of Neurosurgery, Jacobs School of Medicine and Biomedical Sciences at the University at Buffalo, Buffalo NY, USA \\ ${ }^{2}$ Department of Neurosurgery, Buffalo General Medical Center, Kaleida Health, Buffalo NY, USA \\ ${ }_{3}^{3}$ Jacobs School of Medicine and Biomedical Sciences at the University at Buffalo, Buffalo NY, USA \\ Email: *jpollina@ubns.com
}

How to cite this paper: O'Connor, T.E., O'Hehir, M.M., Mao, J.Z., Mullin, J.P. and Pollina, J. (2021) Prone Transpsoas Approach for Adjacent Segment Disease and Flatback Deformity: Technical Note and Case Report. Open Journal of Modern Neurosurgery, 11, 20-28.

https://doi.org/10.4236/ojmn.2021.111003

Received: October 31, 2020

Accepted: December 6, 2020

Published: December 9, 2020

Copyright $\odot 2021$ by author(s) and Scientific Research Publishing Inc. This work is licensed under the Creative Commons Attribution International License (CC BY 4.0).

http://creativecommons.org/licenses/by/4.0/

\begin{abstract}
The prone transpsoas approach is a relatively new technique to correct segmental kyphosis and global sagittal imbalance in a minimally invasive fashion. Here, we provide a detailed case report using the prone transpsoas approach to address adjacent segment disease and flatback deformity. This technique allows considerable restoration of segmental lordosis with lateral interbody placement and posterior decompression and fusion using a single position approach. Our experience with the surgical technique and the advantages and challenges unique to this approach are discussed.
\end{abstract}

\section{Keywords}

Prone Transpsoas, Adjacent Segment Disease, Flatback Deformity

\section{Introduction}

Surgical treatment options have expanded over the last decade to correct adjacent segment disease and global sagittal balance. Traditionally, posterior-only approaches were standard [1]. Ventral approaches to the lumbar spine have become a powerful alternative to posterior-only approaches to restore segmental lordosis. In recent years, lateral lumbar interbody and fusion (LLIF) with posterior hardware placement for stabilization has become a widely used technique [2]. In this approach, patients must be re-positioned intraoperatively or have the operation spread out over several days. The prone transpsoas (PTP) technique is a novel method that allows placement of a lordotic lateral interbody implant with revision of posterior instrumentation in a single-position approach. As the 
patient population ages and surgical techniques advance, minimally invasive options will allow the goals of surgery to be accomplished with decreased hospital lengths of stay and reduced overall complication rates.

\section{Case Presentation}

A 72-year-old male presented with progressive mechanical back pain radiating to his anterolateral thighs 9 years after undergoing an L4-S1 fusion. His back pain and lower-extremity radiculopathy were exacerbated by standing, and he was unable to ambulate more than 50 feet secondary to back pain and neurogenic claudication. His symptoms continued to worsen in spite of 6 months of conservative treatment that included physical therapy and interventional pain management.

His medical history was significant for congestive heart failure, obesity (body mass index of 32), obstructive sleep apnea, diabetes, and hypertension. Computed tomography (CT) imaging demonstrated solid fusion from his previous construct at L4 -S1 and adjacent segment disease (ASD) with lumbar stenosis at L3-4 (Figure 1). Scoliosis standing X-rays revealed a significant positive sagittal imbalance with a sagittal vertical axis (SVA) of $14 \mathrm{~cm}$, segmental kyphosis of approximately $10^{\circ}$, and a $28^{\circ}$ pelvic incidence to lumbar lordosis (PI-LL) mismatch (Figure 2). Written patient consent was obtained before surgery to use clinical information, imaging, and photographs for clinical and educational purposes.

\section{Technical Report}

\subsection{Surgical Plan}

The goals of this patient's surgical procedure were restoration of the PI-LL mismatch to $10^{\circ}$, reduction of the SVA to $+5 \mathrm{~cm}$, decompression at L3-L4, and fusion at the operative level [3]. In the setting of the patient's solid fusion from L4-S1, ASD with stenosis, segmental kyphosis at L3-L4, and multiple comorbidities, a more minimally invasive approach was considered. A single-position prone transpsoas (PTP) approach was planned to restore segmental lordosis, decrease the PI-LL mismatch, correct the patient's global sagittal imbalance, and reduce the total operative time.

\subsection{Surgical Procedure}

The patient was turned to a prone position on a Jackson table and secured using specialized PTP bolsters (Figure 3). Neuromonitoring electrodes (SafeOp Surgical, Inc., Hunt Valley, Maryland) were placed, and electromyographic findings were monitored at baseline and throughout the case. The Jackson table was then raised approximately 5 feet off the floor to eye level. The L3-4 disk space was identified by rotating the fluoroscopy machine until orthogonal anteroposterior (AP) and lateral views were obtained (Figure 4). An incision was made in the left flank, and the retroperitoneal transpsoas approach to the L3-4 disk space was 
carried out in standard fashion. During the approach and deployment of the retractor (Alphatec Spine, Carlsbad, California), the neuromonitoring recordings were satisfactory at $19 \mathrm{~mA}$. The retractor was deployed, and the disk space at L3-4 was exposed (Figure 5). An annulotomy was performed, and the discectomy was completed. An IdentiTI porous titanium interbody implant (Alphatec Spine) was then placed ( $9 \mathrm{~mm}$ anterior height, $22 \mathrm{~mm}$ width, $50 \mathrm{~mm}$ length, and $15^{\circ}$ lordosis) (Figure 6, Figure 7).
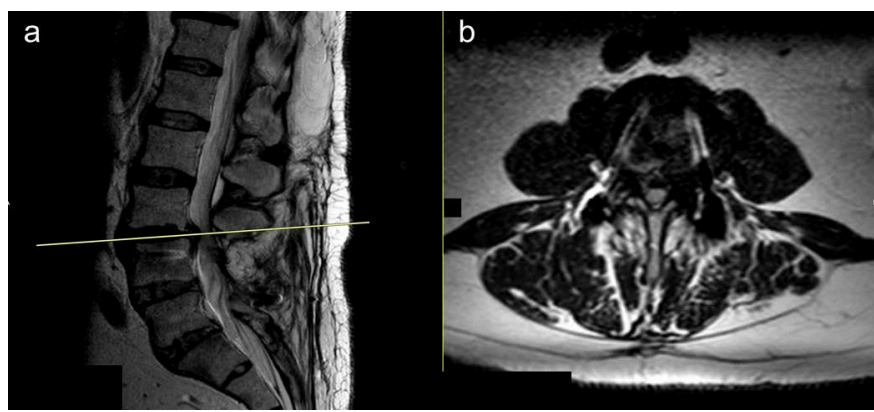

Figure 1. Magnetic resonance images of the lumbar spine demonstrate single-level lumbar stenosis at L3-4 above the previous fusion construct at L4-S1 ((a) sagittal; (b) axial).

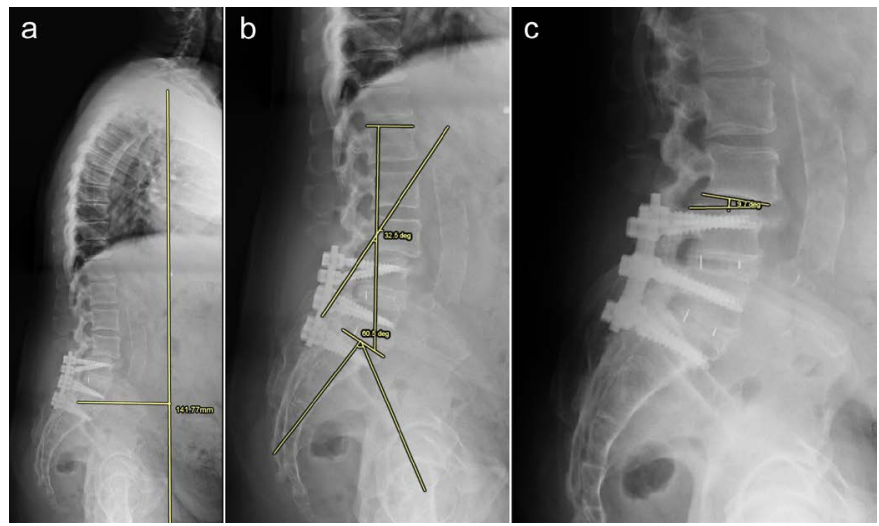

Figure 2. Standing X-rays demonstrate positive sagittal imbalance with a sagittal vertical axis (SVA) of $+14 \mathrm{~cm}$ (a) and a pelvic incidence of $60^{\circ}$ with lumbar lordosis of $32^{\circ}$ (b), and segmental kyphosis of approximately $10^{\circ}$ (c) at L3-4.

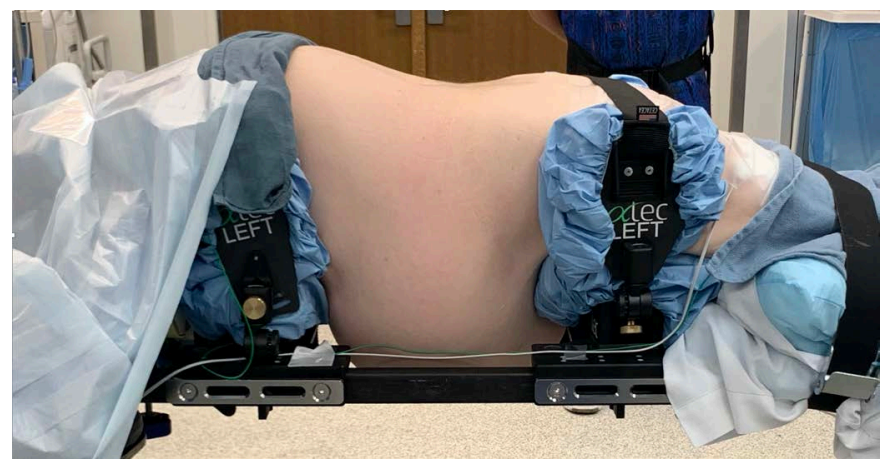

Figure 3. Operative photograph shows the patient positioned for the prone transpsoas (PTP) approach. Specially designed bolsters leave the abdomen free hanging and allow rotation of the pelvis to lower the iliac crest for access. 


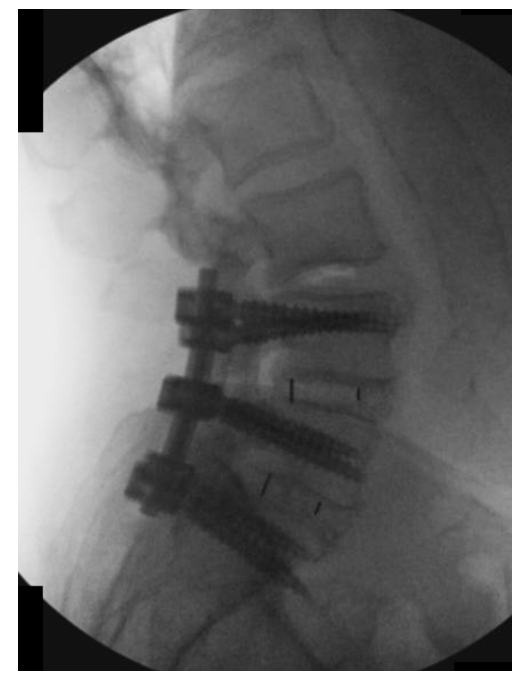

Figure 4. Sagittal X-ray obtained after positioning the patient prone within the PTP bolsters. With positioning alone, considerable restoration of lordosis is noted compared to the preoperative standing X-rays in Figure 2.

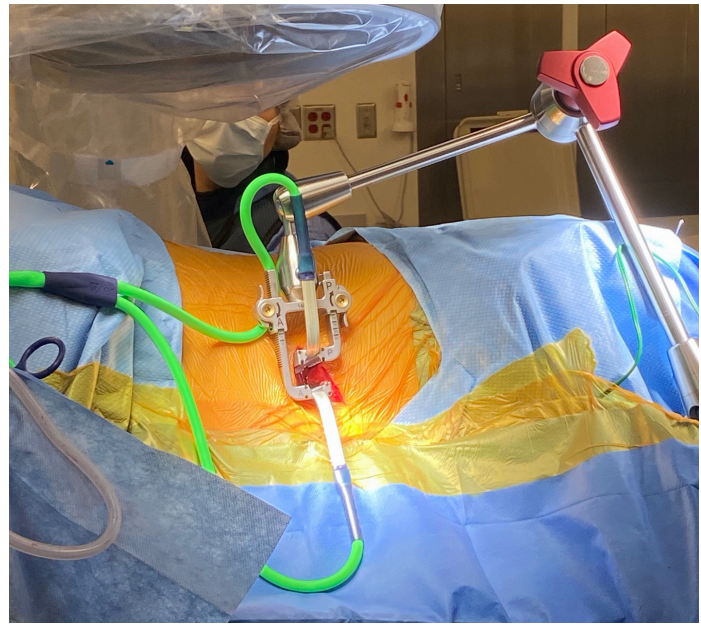

Figure 5. Operative photograph shows the rigid retractor system with anterior and posterior blades (Alphatec Spine, Carlsbad, California) that is used for the PTP approach.
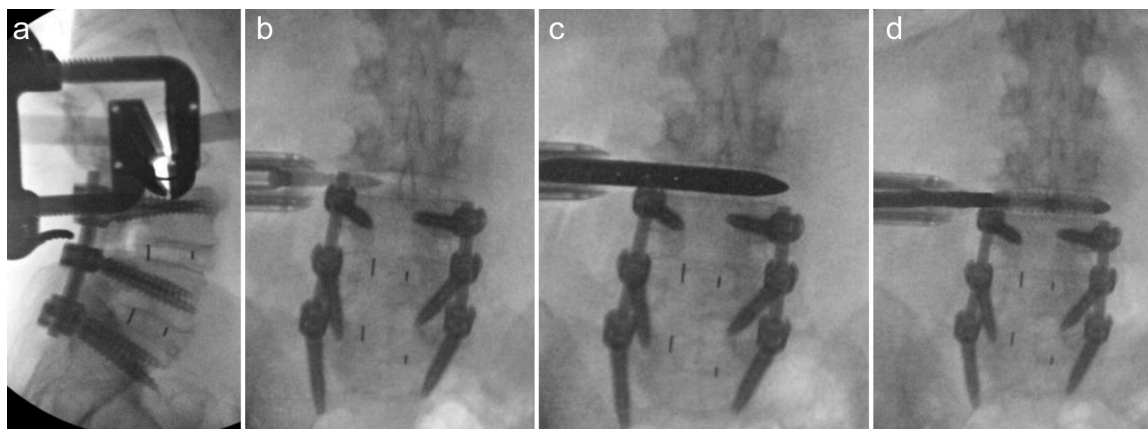

Figure 6. Lateral X-rays demonstrate placement of the PTP retractor at the disc space (a). Anteroposterior (AP) X-ray demonstrates deployment of the shim in the L3-4 disc space (b). A trial interbody implant is temporarily placed prior to insertion of the final interbody implant (c). An IdentiTI porous titanium interbody implant (Alphatec Spine) is placed with $9 \mathrm{~mm}$ anterior height, $22 \mathrm{~mm}$ width, $50 \mathrm{~mm}$ length, and $15^{\circ}$ of lordosis (d). 


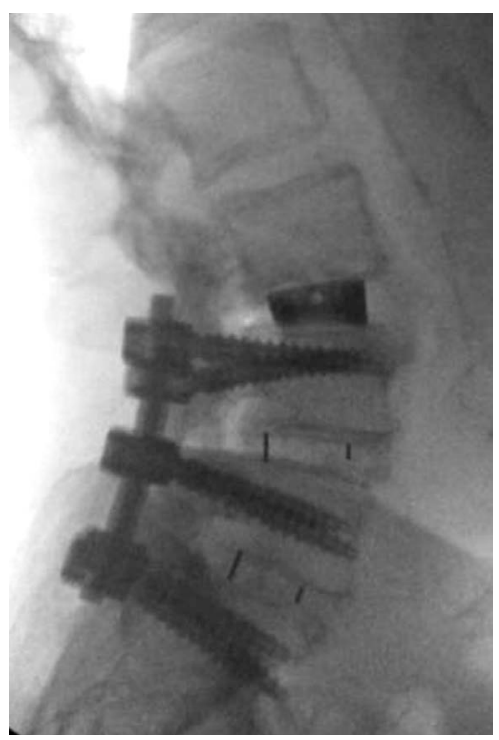

Figure 7. Lateral X-ray demonstrating placement of the $15^{\circ}$ lordotic titanium interbody implant at L3-4.

While keeping the operative field sterile with the patient draped in the prone position, the posterior instrumentation was then placed in a single position (Figure 8). Bilateral posterior pedicle screws were placed at L3 and secured to the prior posterior construct (Figure 9, Figure 10).

\section{Results}

Following placement of the lateral interbody implant and posterior instrumentation, segmental lordosis increased by $20^{\circ}$ at the operative level, from $10^{\circ}$ of kyphosis to $10^{\circ}$ of lordosis (Figure 11). Postoperatively, the PI-LL mismatch decreased from $28^{\circ}$ to $10^{\circ}$ (Figure 11), and the sagittal imbalance was significantly improved from $+14 \mathrm{~cm}$ to $+5 \mathrm{~cm}$ (Figure 11). The patient did well in the immediate postoperative period. He reported improvement in his preoperative symptoms and was discharged home on postoperative day 2 .

\section{Discussion}

The PTP approach offers several advantages to the more traditional lateral position that is typically used for transpsoas lumbar interbody fusion [4] [5]. The prone positioning has the potential to improve segmental lordosis and increase operative efficiency by facilitating placement of the lateral interbody implant and posterior instrumentation.

This technique shows promise in treating ASD in pre-existing posterior spinal fusions [6]. In the prone position with the abdomen free-hanging, positioning alone can use gravity to the surgeon's advantage and significantly increase lordosis at the operative level (Figure 4). The lateral interbody implant can be placed to increase segmental lordosis and achieve indirect decompression [7].

The specialized PTP bolsters can rotate the pelvis and lower the iliac crest to allow increased access to the disc space. This is particularly helpful when per- 
forming PTP approaches at the L4-5 level to obviate the need for angled instruments (Figure 12).

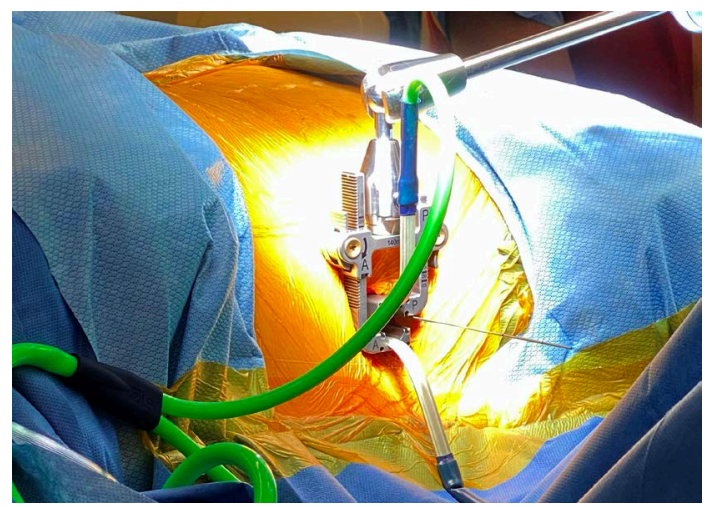

Figure 8. Operative photograph demonstrates the position and draping of the surgical field, allowing the lateral and posterior operations to be completed in a single position.

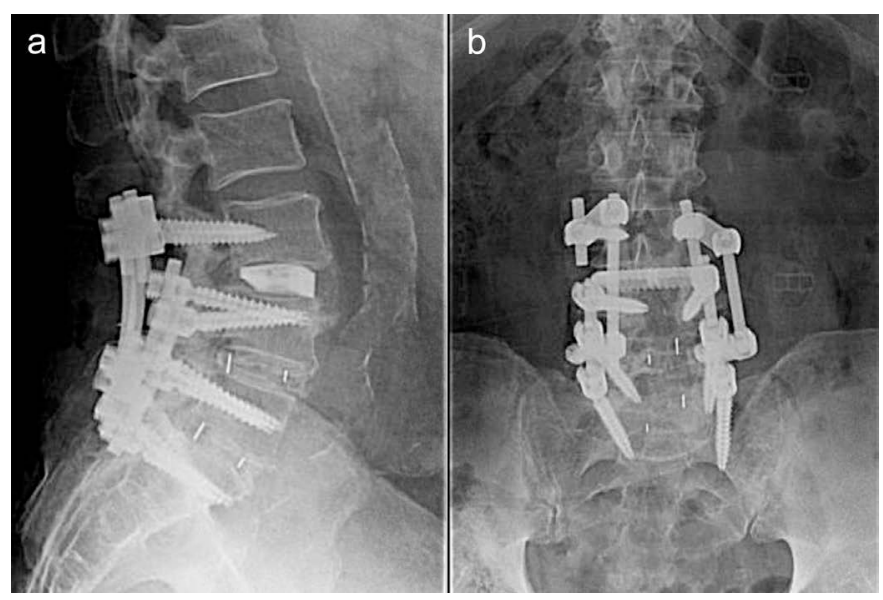

Figure 9. Lateral (a) and AP (b) X-rays demonstrate final placement of the L3-4 instrumentation. The short rods noted at L3 are "orphan" rods that are necessary when using the current dual tulip-head design of the screw head.

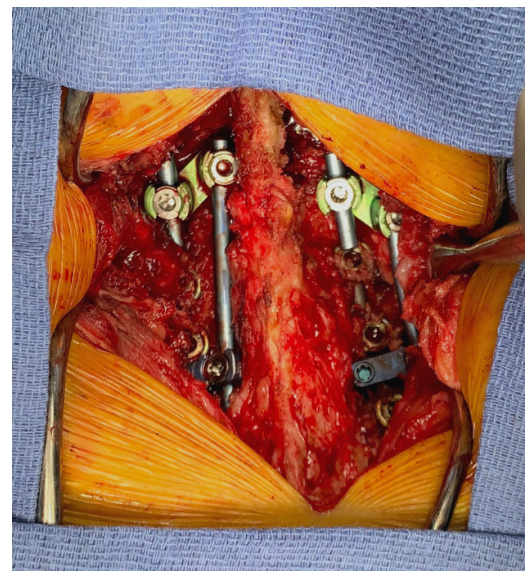

Figure 10. Operative photograph shows the L3 pedicle screws attached to the previous posterior construct. "Orphan" rods were used for final tightening of the dual tulip-head screw heads. 


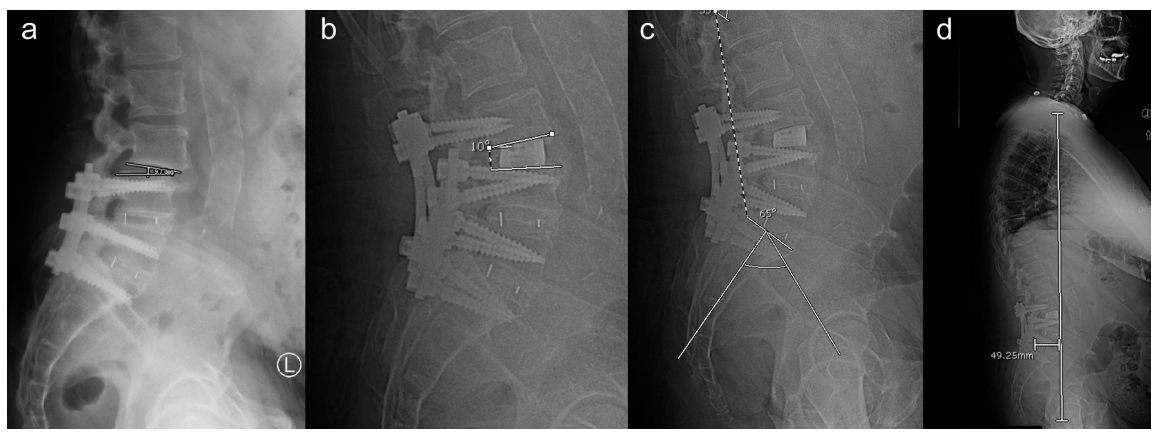

Figure 11. Postoperative lateral X-ray demonstrates significant increase of segmental lordosis at L3-4 from approximately $10^{\circ}$ of kyphosis in the preoperative $\mathrm{x}$-ray (a) to $10^{\circ}$ of lordosis postoperatively (b). Postoperative X-ray demonstrates an increase in lumbar lordosis from $32.5^{\circ}$ preoperatively to $55^{\circ}$ postoperatively (c). The pelvic incidence to lumbar lordosis (PI-LL) mismatch is decreased from $28^{\circ}$ preoperatively to $10^{\circ}$ postoperatively. Postoperative standing lateral X-ray demonstrates correction of global sagittal imbalance from $+14 \mathrm{~cm}$ preoperatively to $+5 \mathrm{~cm}$ postoperatively (d).

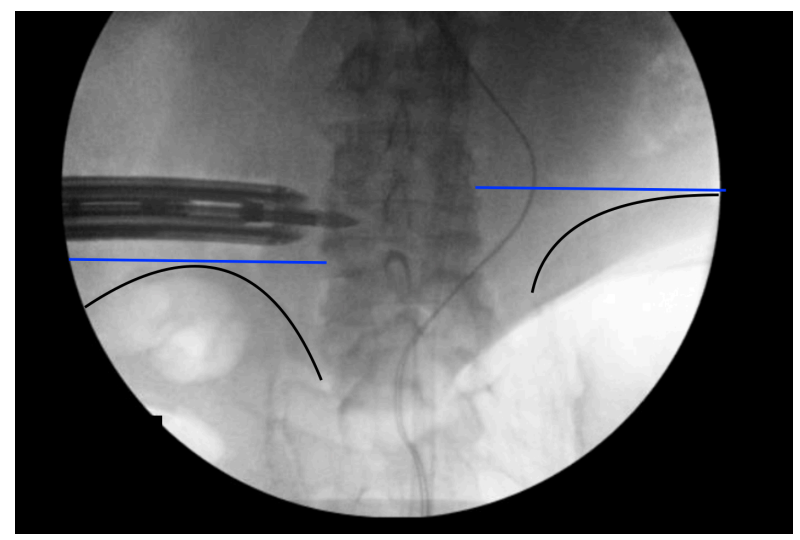

Figure 12. Lateral X-ray demonstrates the ability of the PTP bolsters to lower the iliac crest to allow placement of the retractor system and potentially obviate the need for angled instruments at the L4-5 interspace. The black line outlines the top of the iliac crest and the blue line demonstrates the disc space that can be accessed at the top of the crest.

Another advantage of the PTP approach is the ability to insert lateral and posterior instrumentation in a single position [8]. While maintaining a sterile operative field with the patient draped in the prone position, the posterior instrumentation can then be placed immediately after lateral interbody implant placement. In some cases, the posterior incision can be made and posterior instrumentation can be exposed simultaneously before the conclusion of the lateral portion of the case. This saves considerable time in the operating room by eliminating the need to flip the patient and the time associated with additional positioning [9]. The instrumentation used in this technique may also be used in other approaches and does not contribute to any added cost of the procedure. The only potential for added cost is the use of the specialized bolsters; however, this cost should be weighed against the potential for notable cost reduction by decreasing operative time.

This novel approach requires certain considerations. There is a learning curve, 
as associated with any new surgical procedure; however, the principles governing the transpsoas technique remain the same. There may be surgeon discomfort while operating at a higher working height, with certain maneuvers performed with outstretched arms at standing height. The effect of gravity is advantageous for increasing lumbar lordosis while simultaneously causing soft tissue sag. To mitigate soft tissue sagging, the incision may be made slightly posterior to the typical approach. Finally, the height of the iliac crest can be a limiting factor to access the more caudal disc spaces. This is a common challenge with lateral approaches, though, and the unique bolster used in this case study allow for rotation of the pelvis to drop the iliac crest and assist with access to the desired disc space.

\section{Conclusion}

The prone-transpsoas technique allowed for significant restoration of segmental lordosis in a single position approach. This case illustrates the advantages of using this approach to correct flat back deformity with a relatively minimally invasive approach in order to achieve significant correction of global sagittal imbalance.

\section{Acknowledgements}

The authors thank Paul H. Dressel for formatting the images and Sharon O'Connor and Debra J. Zimmer for editorial assistance with this manuscript.

\section{Funding}

This research received no specific grant from any funding agency in the public, commercial, or not-for-profit sectors.

\section{Ethics}

At the time of hospital admission, informed consent for patient information to be published was provided by the patient. The University at Buffalo institutional review board does not require approval for a technical note-case report involving a single patient.

\section{Author Contributions}

Conceptualization: JP, TEO; Data curation: JP, TEO; Formal analysis: All authors; Funding acquisition: Not applicable; Investigation: JP, TEO; Methodology: JP, TEO Project administration: JP, TEO; Supervision: JP, TEO; Roles/Writing original draft: TEO, MMO; Writing review \& editing: All authors.

\section{Financial Relationships}

TEO receives research funding from the AOSpine Foundation as part of the Discovery and Innovation Award.

MMO: No financial relationships to disclose. 
JZM receives research funding from AOSpine North America (AOSNA) for works to advance $3 \mathrm{D}$ printing.

JPM is involved with clinical research for Cerapedics. He receives research funding from AOSNA and the Research Committee Award \#87639 and from Medtronic External Research Program Health Professionals, ERP ID\#2020-12271.

JP is involved with surgical training for Medtronic and serves as a consultant for and receives royalties from ATEC Spine.

\section{Conflicts of Interest}

The authors declare no conflicts of interest regarding the publication of this paper.

\section{References}

[1] Daffner, S.D. and Vaccaro, A.R. (2003) Adult Degenerative Lumbar Scoliosis. American journal of orthopedics (Belle Mead N), 32, 77-82. https://doi.org/10.1007/s004820100036

[2] Attenello, J., Chang, C., Lee, Y.P., Zlomislic, V., Garfin, S.R. and Allen, R.T. (2018) Comparison of Lateral Lumbar Interbody Fusion (LLIF) with Open versus Percutaneous Screw Fixation for Adult Degenerative Scoliosis. Journal of Orthopaedics, 15, 486-489. https://doi.org/10.1016/j.jor.2018.03.017

[3] Le Huec, J.C., Thompson, W., Mohsinaly, Y., Barrey, C. and Faundez, A. (2019) Sagittal Balance of the Spine. European Spine Journal, 28, 1889-1905. https://doi.org/10.1007/s00586-019-06083-1

[4] Allain, J. and Dufour, T. (2020) Anterior Lumbar Fusion Techniques: ALIF, OLIF, DLIF, LLIF, IXLIF. Orthopaedics \& Traumatology: Surgery \& Research, 106, S149-S157. https://doi.org/10.1016/j.otsr.2019.05.024

[5] Zhang, T., Bai, S., Dokos, S., Cheung, J.P. and Diwan, A.D. (2019) XLIF Interbody Cage Reduces Stress and Strain of Fixation in Spinal Reconstructive Surgery in Comparison with TLIF Cage with Bilateral or Unilateral Fixation: A Computational Analysis. 201941 st Annual International Conference of the IEEE Engineering in Medicine and Biology Society (EMBC), Berlin, 23-27 July 2019, 1887-1890. https://doi.org/10.1109/EMBC.2019.8856592

[6] Shasti, M., Koenig, S.J., Nash, A.B., et al. (2019) Biomechanical Evaluation of Lumbar Lateral Interbody Fusion for the Treatment of Adjacent Segment Disease. The Spine Journal, 19, 545-551. https://doi.org/10.1016/j.spinee.2018.09.002

[7] Taba, H.A. and Williams, S.K. (2020) Lateral Lumbar Interbody Fusion. Neurosurgery Clinics of North America, 31, 33-42. https://doi.org/10.1016/j.nec.2019.08.004

[8] Godzik, J., Ohiorhenuan, I.E., Xu, D.S., et al. (2020) Single-Position Prone Lateral Approach: Cadaveric Feasibility Study and Early Clinical Experience. Neurosurgical Focus, 49, E15. https://doi.org/10.3171/2020.6.FOCUS20359

[9] Lamartina, C. and Berjano, P. (2020) Prone Single-Position Extreme Lateral Interbody Fusion (Pro-XLIF): Preliminary Results. European Spine Journal, 29, 6-13. https://doi.org/10.1007/s00586-020-06303-Z 